\title{
Connective tissue growth factor (CCN2, CTGF) and organ fibrosis: lessons from transgenic animals
}

\author{
David R. Brigstock
}

Received: 4 September 2009 /Accepted: 13 September 2009/Published online: 2 October 2009

(C) The Author(s) 2009. This article is published with open access at Springerlink.com

\begin{abstract}
In recent months, four different systems have been reported in the literature in which CCN2 transgenes were individually expressed in podocytes, hepatocytes, cardiomyocytes or respiratory epithelial cells to achieve overexpression in, respectively, the kidney, liver, heart, or lung. These transgenic systems have provided valuable information about the contribution of CCN2 to fibrosis in vivo and have begun to reveal the complexities of the underlying mechanisms involved. On the one hand, studies of these animals have revealed that $\mathrm{CCN} 2$ overexpression does not necessarily lead directly to fibrotic pathology but may cause severe non-fibrotic tissue damage due to its other effects on cell function (e.g. heart). On the other hand, overexpression of CCN2 in concert with signaling pathways associated with development (e.g. lung) or fibrosing injuries (e.g. kidney, liver) can lead to the initiation or exacerbation of fibrosis. The significance of these studies is discussed in the context of the requirement for interactions between $\mathrm{CCN} 2$ and co-stimulatory factors in the microenvironment for the manifestation of CCN2dependent fibrosis.
\end{abstract}

Keywords CCN2 - Connective tissue growth factor C CTGF . Fibrogenesis $\cdot$ Matricellular $\cdot$ Transgene

\footnotetext{
D. R. Brigstock ( $\bowtie)$

Center for Clinical and Translational Research,

The Research Institute, Nationwide Children's Hospital,

Room WA2022, 700 Children's Drive,

Columbus, OH 43205, USA

e-mail: David.Brigstock@NationwideChildrens.Org

D. R. Brigstock

Departments of Surgery and Molecular and Cellular Biochemistry,

The Ohio State University,

Columbus, OH 43212, USA
}

\begin{abstract}
Abbreviations
CCN2 (CTGF) Connective tissue growth factor

TGF- $\beta$ Transforming growth factor beta
\end{abstract}

\section{Introduction}

For well over a decade, the association of connective tissue growth factor (CCN2, also known as CTGF) with fibrotic diseases has been the driving force behind many investigations designed to understand its biology and biochemistry. There are now countless studies that attest to the importance of this molecule in fibrotic pathology. Indeed, the initial recognition of $\mathrm{CCN} 2$ as a downstream mediator of the pro-fibrogenic effects of transforming growth factor beta (TGF- $\beta$ ) has resulted in elegant investigations of the transcriptional regulation of the $\mathrm{CCN} 2$ gene as well as to its recognition as a potentially superior anti-fibrotic target (Leask et al. 2009). Extensive investigations have shown convincingly that CCN2 expression is up-regulated in fibrotic disorders, that it is produced temporally and spatially in close proximity to fibrotic areas in vivo, and that it is often localized to and a target for fibrotic (procollagenic) cells (Leask et al. 2002; Rachfal and Brigstock 2005). Antagonists of $\mathrm{CCN} 2$ have proven effective in blocking pro-fibrogenic $\mathrm{CCN} 2$ signaling pathways in vitro and have yielded promising data with respect to preventing or reversing fibrosis in several animal models in vivo (Brigstock 2009).

What more, then, do we need to know? Unfortunately, as with many other areas of CCN2 research, the devil is in the details. The recognition of $\mathrm{CCN} 2$ as a matricellular protein rather than a conventional growth factor (Rachfal and Brigstock 2005; Leask and Abraham 2006; Chen and Lau 
2009) requires us to think differently as to how this molecule is involved in fibrosis. Collectively, its multidomain structure, association with the extracellular matrix, utilization of multiple receptors on the cell surface (integrins, heparan sulfate proteoglycans, low density lipoprotein receptor-related protein, nerve growth factor tyrosine kinase receptor), binding interactions with other cell-regulatory molecules (TGF- $\beta$, insulin-like growth factor, vascular endothelial growth factor), and propensity for proteolytic cleavage into various bioactive fragments adds many layers of complexity to its mode of action and we have either a very rudimentary understanding or no knowledge at all as to the importance of these or other factors in determining the fibrotic response to CCN2. Nonetheless, for many years there has been a dogma that "CCN2 drives fibrosis". Is this accurate? Data reported over the last few months regarding some new and very interesting $\mathrm{CCN} 2$ transgenic models have now begun to shed light on this critical issue. These studies have revealed that the consequences of CCN2 overexpression are not necessarily predictable and that the manifestation of $\mathrm{CCN} 2$ fibrotic activity in vivo involves crucial interactions with co-stimulatory signals in the microenvironment.

\section{Transgenic systems for CCN2}

Four different systems have recently been described in which CCN2 transgenes were individually expressed in podocytes (Yokoi et al. 2008), hepatocytes (Tong et al. 2009), cardiomyocytes (Panek et al. 2009) or respiratory epithelial cells (Wu et al. 2009) to achieve overexpression in, respectively, the kidney, liver, heart, or lung. Kidneyoverexpressing transgenic $\mathrm{C} 57 \mathrm{BL} / 6 \mathrm{~J}$ mice constitutively expressed a mouse $\mathrm{CCN} 2$ transgene that was under the control of a human nephrin promoter; mice were examined up to 24 weeks of age (Yokoi et al. 2008). Liveroverexpressing transgenic $\mathrm{FVB} / \mathrm{n}$ mice were produced in this laboratory and constitutively expressed a human CCN2 transgene that was under the control of a mouse albumin promoter enhancer; mice were examined up to 28 weeks of age (Tong et al. 2009). Heart-overexpressing FVB/n mice or Sprague-Dawley rats constitutively expressed rat CCN2 under the control of a mouse myosin light chain -2 promoter; animals were examined up to 7 months of age (Panek et al. 2009). Lung-overexpressing transgenic C57BL/6 J mice conditionally expressed human CCN2 under the control of the Clara cell secretary protein promoter using a deoxycycline-inducible system; transgene expression was induced over the first 2 weeks of post-natal life at which time the mice were examined (Wu et al. 2009).

Directed overexpression of the CCN2 transgene was successfully documented in all of these systems. However, the most surprising observation was that $\mathrm{CCN} 2$ overexpression in the kidney, liver, or heart consistently failed to cause any kind of fibrotic reaction whatsoever. Kidney transgenics exhibited no glomerular abnormalities or proteinuria while liver transgenics exhibited no hepatic histological abnormalities or elevated liver function tests. In contrast, the heart transgenics exhibited cardiomyocyte hypertrophy and age-dependent heart disease which progressed from compensatory hypertrophy to ventricular dilation and systolic heart failure - but even so these dysfunctional hearts were not fibrotic. On the other hand, the lung transgenic mice provided clear evidence that CCN2 overexpression during the first 2 weeks of postnatal life causes abnormal alveolarization and impaired formation of the alveolar vascular network and that these deficiencies were associated with fibrosis in and around the alveolar septa, bronchi, bronchioles, and vessels.

How do we reconcile these findings? One possibility is that the pro-fibrotic effects of CCN2 may be dependent on the developmental stage of the target organ. Perhaps mere overexpression of CCN2 in the kidney, liver or heart was insufficient to drive fibrosis because these organs were examined in mature animals whereas the lung transgenics were analyzed at an early and critical time of lung development when, possibly, the transgenic CCN2 protein was able to interact with and somehow impair essential growth, differentiation or maturation signaling pathways. If this is the case, one might speculate that the pathological sequelae of pulmonary CCN2 overexpression might be less severe in more mature adult animals, a question that is highly suitable to the inducible transgenic system developed for these mice. Likewise, it also would be interesting to assess fibrosis during critical stages of organ development in the kidney, liver and heart transgenics, although if any such abnormality was present during pre- or early postnatal life it appears to be resolved by adulthood.

The story does not stop there, however. Interesting additional data were reported for the kidney and liver transgenics that provide important clues as to the role of CCN2 in fibrotic pathways. When the kidney transgenic mice were challenged with streptozotocin to induce type I diabetes, they exhibited a more pronounced glomerular pathology than wild-type littermates in as much as they demonstrated mesangial expansion, podocyte loss, and a reduction in the activity of matrix-metalloprotease 2 , a matrix-degrading enzyme that is often suppressed in fibrotic conditions. Thus the effect of the $\mathrm{CCN} 2$ transgene was to exacerbate some aspects of a fibrotic phenotype in a setting of diabetic nephropathy, although expression of extracellular matrix components such as fibronectin or collagen was unchanged and no fibrosis per se was observed (Yokoi et al. 2008). A somewhat similar but more compelling scenario was observed in our liver transgenic 
mice after they were subjected to liver injury by carbon tetrachloride administration or ligation of the bile duct (Tong et al. 2009). Both of these interventions are wellcharacterized methods of inducing hepatic fibrosis yet the transgenic mice fared worse than their wild-type littermates as shown by the presence of more $\alpha$-smooth muscle actinpositive cells (presumptive activated hepatic stellate cells, a principal pro-fibrogenic cell type in the liver), increased deposition of collagen, and elevated mRNA expression of key fibrotic markers including $\alpha$-smooth muscle actin, collagen $\alpha 1(\mathrm{I})$, and tissue inhibitor of matrix-metalloprotease-1 (the latter of which blocks matrix breakdown and favors collagen accumulation). This phenomenon was highly reproducible as further exemplified by the additional data shown in Fig. 1 which illustrates that the liver transgenic mice treated chronically with the hepatotoxin thioacetamide show a greatly exaggerated fibrotic response in their livers as compared to their wild-type counterparts. We have further shown that homozygous liver transgenic mice exhibit more severe hepatic fibrosis than heterozygous liver transgenic mice in response to carbon tetrachloride (Tong et al. 2009). This finding is important because it suggests that CCN2 transgene dose may also account for some of the phenotypic variability reported between the four transgenic systems, a question that will require clarification through further experimental analysis.

Collectively, the observations to date support the notion that the pro-fibrogenic properties of CCN2 are highly contextual. Whereas normal adult tissues are seemingly refractory to elevated CCN2 levels (at least in terms of the fibrotic response), the pro-fibrotic properties of CCN2 are manifested in conjunction with other changes in the microenvironment, such as those induced by tissue damage or during wound healing. The co-stimulatory signals that contribute to this phenomenon may include known synergistic factors such as TGF- $\beta$ or fibronectin (Tong et al. 2009), changes in the level or nature of signaling through CCN2 receptors, components of additional signaling pathways in injured tissues such as those associated with inflammation, necrosis or wound healing, or modifications to the structure or composition of the extracellular matrix which is a reservoir of many bioactive molecules including CCN2 itself. In addition, injured tissues contain infiltrating immune cells and macrophages as well as specialized cells that promote wound healing, and the presence of these cells may be important either directly or indirectly for CCN2 fibrotic action. However, data from the heart transgenic animals show that pathways of tissue damage or pathology that are themselves CCN2-dependent do not necessarily cause co-stimulatory pro-fibrogenic pathways to be triggered. Further, since different strains of mice show variable responses to common fibrotic agents (Schrier et al. 1983; Hillebrandt et al. 2002; Bonniaud et al. 2004) as well as in production of CCN2 itself (Cardoso et al. 2009), genetic background likely plays an important role in influencing the fibrotic action of CCN2.
Fig. 1 Enhanced thioacetamideinduced hepatic fibrosis in $\mathrm{CCN} 2$ transgenic livers. CCN2 $\mathrm{FVB} / \mathrm{n}$ transgenic mice were produced as described (Tong et al. 2009). Four-week old wildtype $(\mathbf{a}, \mathbf{b})$ or homozygous transgenic $(\mathbf{c}, \mathbf{d})$ mice $(n=4$ per group) were treated three times a week with thioacetamide (200 mg/kg i.p.) for four weeks. Liver sections were stained immunohistochemically for $\alpha$ smooth muscle actin (a, c; brown staining) or with Sirius Red for collagen deposition (b, d; red staining). Staining is shown at $10 \times$
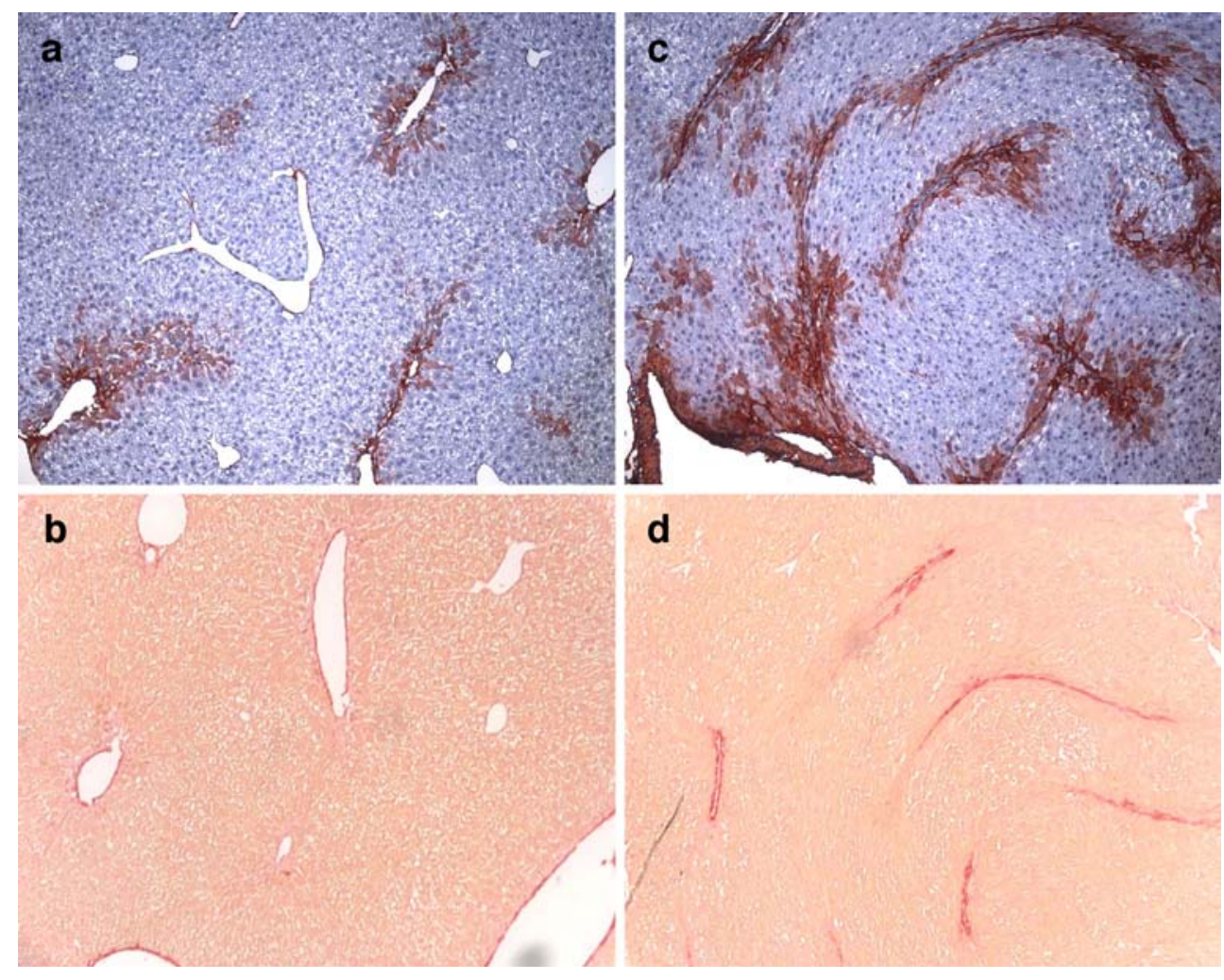

d

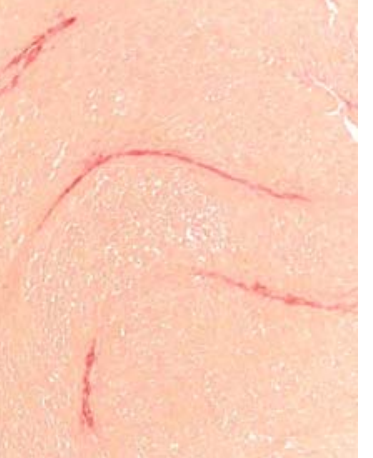


The concept of a requirement for co-stimulants may well prove to be the unifying feature in these animal models in that elevated CCN2 levels are not fibrotic (e.g. kidney, liver, heart) unless there is a background of other signaling events produced by processes such as growth and development (e.g. lung) or injury and wound healing (e.g. kidney, liver). Indeed, the data obtained from the CCN2 transgenic animals reinforce previous in vivo studies which have pointed to a modifying rather than causative role for $\mathrm{CCN} 2$ in fibrosis. For example, subcutaneous co-administration of TGF- $\beta$ and $\mathrm{CCN} 2$ resulted in protracted and sustained dermal fibrosis whereas this effect was not achieved by either TGF- $\beta$ or CCN2 individually (Mori et al. 1999). Additionally, Balb/c mice are genetically resistant to bleomycin-induced lung fibrosis but nonetheless developed fibrosis after administration of bleomycin and a CCN2-overexpressing adenovirus (Bonniaud et al. 2004). The behavior of CCN2 in all of these studies is likely illustrative of its role as a matricellular protein whereby it resides in the matrix or is tethered to the cell surface and modifies cellular function by 'sensing' changes in the environment through its engagement of a myriad of molecular binding partners. CCN2 has been likened to a molecular hub or adapter (Leask and Abraham 2006), capable of integrating inputs from multiple signaling pathways and regulating cellular responses accordingly. Thus, 'readouts' for CCN2 are highly dependent on a plethora of other molecular cues within the microenvironment and conclusions about its activity - including its role in fibrosis - cannot be made without careful attention to contextual considerations. In turn, this raises broader questions such as how to best design meaningful mechanistic studies to probe CCN2 action (especially those that rely on in vitro approaches) and whether components of its co-stimulatory pathways are useful targets for fibrotic therapy in vivo.

In conclusion, $\mathrm{CCN} 2$ transgenic systems have begun to provide valuable information about the fibrotic pathways that may be triggered by $\mathrm{CCN} 2$ overexpression in vivo. On the one hand, overexpression of CCN2 does not necessarily lead directly to fibrotic pathology (e.g. kidney, liver, heart) but can cause severe non-fibrotic tissue damage due to other deleterious effects on cell function (e.g. heart). On the other hand, overexpression of CCN2 in concert with signaling pathways associated with growth and development (e.g. lung) or fibrosing injuries (e.g. kidney, liver) can lead to the initiation or exacerbation of fibrosis.

So, does CCN2 drive fibrosis? Sometimes yes. Sometimes no. Context is the key.

Acknowledgments This work was supported by NIH grants AA012817 and AA016003. I recognize all of the members of my research team for their resilience in grappling with the complexities of CCN2 biology on a day-to-day basis. I am especially indebted to Ruju Chen for generating the data shown in Fig. 1.
Open Access This article is distributed under the terms of the Creative Commons Attribution Noncommercial License which permits any noncommercial use, distribution, and reproduction in any medium, provided the original author(s) and source are credited.

\section{References}

Bonniaud P, Martin G, Margetts PJ, Ask K, Robertson J, Gauldie J, Kolb M (2004) Connective tissue growth factor is crucial to inducing a profibrotic environment in "fibrosis-resistant" BALB/ c mouse lungs. Am J Respir Cell Mol Biol 31:510-516

Brigstock DR (2009) Strategies for blocking the fibrogenic actions of connective tissue growth factor (CCN2): from pharmacological inhibition in vitro to targeted siRNA therapy in vivo. J Cell Commun Signal 3:5-18

Cardoso JF, Mendes FA, Amadeu TP, Romana-Souza B, Valenca SS, Porto LC, Abreu JG, Monte-Alto-Costa A (2009) Ccn2/Ctgf overexpression induced by cigarette smoke during cutaneous wound healing is strain dependent. Toxicol Pathol 37:175-182

Chen CC, Lau LF (2009) Functions and mechanisms of action of CCN matricellular proteins. Int J Biochem Cell Biol 41:771-783

Hillebrandt S, Goos C, Matern S, Lammert F (2002) Genome-wide analysis of hepatic fibrosis in inbred mice identifies the susceptibility locus Hfib1 on chromosome 15. Gastroenterology 123:2041-2051

Leask A, Abraham DJ (2006) All in the CCN family: essential matricellular signaling modulators emerge from the bunker. $\mathrm{J}$ Cell Sci 119:4803-4810

Leask A, Holmes A, Abraham DJ (2002) Connective tissue growth factor: a new and important player in the pathogenesis of fibrosis. Curr Rheumatol Rep 4:136-142

Leask A, Parapuram SK, Shi-Wen X, Abraham DJ (2009) Connective tissue growth factor (CTGF, CCN2) gene regulation: a potent clinical bio-marker of fibroproliferative disease? J Cell Commun Signal 3:89-94

Mori T, Kawara S, Shinozaki M, Hayashi N, Kakinuma T, Igarashi A, Takigawa M, Nakanishi T, Takehara K (1999) Role and interaction of connective tissue growth factor with transforming growth factor-beta in persistent fibrosis: a mouse fibrosis model. J Cell Physiol 181:153-159

Panek AN, Posch MG, Alenina N, Ghadge SK, Erdmann B, Popova E, Perrot A, Geier C, Morano RD, Bader M, Ozcelik C (2009) Connective tissue growth factor overexpression in cardiomyocytes promotes cardiac hypertrophy and protection against pressure overload. PLoS One 4:e6743

Rachfal AW, Brigstock DR (2005) Structural and functional properties of CCN proteins. Vitam Horm 70:69-103

Schrier DJ, Kunkel RG, Phan SH (1983) The role of strain variation in murine bleomycin-induced pulmonary fibrosis. Am Rev Respir Dis 127:63-66

Tong Z, Chen R, Alt DS, Kemper S, Perbal B, Brigstock DR (2009) Susceptibility to liver fibrosis in mice expressing a connective tissue growth factor transgene in hepatocytes. Hepatology 50:939-947

Wu S, Platteau A, Chen S, McNamara G, Whitsett J, Bancalari E (2009) Conditional over-expression of connective tissue growth factor disrupts postnatal lung development. Am J Respir Cell Mol Biol, Jun 18. [Epub ahead of print] doi:10.1165/rcmb.200900680C

Yokoi H, Mukoyama M, Mori K, Kasahara M, Suganami T, Sawai K, Yoshioka T, Saito Y, Ogawa Y, Kuwabara T, Sugawara A, Nakao $\mathrm{K}$ (2008) Overexpression of connective tissue growth factor in podocytes worsens diabetic nephropathy in mice. Kidney Int $73: 446-455$ 